\title{
Effects of Excitation Density on the Scintillation Properties of Organic-Inorganic Layered Perovskite-type Compounds
}

\author{
Masanori Koshimizu, ${ }^{1 *}$ Naoki Kawano, ${ }^{2}$ Atsushi Kimura, ${ }^{3}$ Satoshi Kurashima, ${ }^{3}$ \\ Mitsumasa Taguchi, ${ }^{3}$ Yutaka Fujimoto, ${ }^{1}$ and Keisuke Asai ${ }^{1}$ \\ ${ }^{1}$ Department of Applied Chemistry, Graduate School of Engineering, Tohoku University, \\ 6-6-07 Aoba, Aramaki, Aoba-ku, Sendai 980-8579, Japan \\ ${ }^{2}$ Akita University, 1-1 Tegata Gakuen, Akita 010-8502, Japan \\ ${ }^{3}$ Takasaki Advanced Radiation Research Institute, National Institutes for Quantum and Radiological Science \\ and Technology, 1233 Watanuki, Takasaki, Gunma 370-1292, Japan
}

(Received January 27, 2021; accepted April 12, 2021)

Keywords: scintillator, linear energy transfer, layered perovskite, Wannier exciton, quantum well

The scintillation properties of organic-inorganic layered perovskite-type compounds were analyzed using pulsed beams having different linear energy transfers (LETs). Initially the decay was slower and then became faster at higher LETs. A possible cause of the slower decay at higher LETs is the competition between the radiative process and trapping at nonradiative sites, resulting in some excitons not being trapped at nonradiative sites at which other excitons have already been trapped at higher LETs. The faster decay at higher LETs is attributed to the interaction of excited states, such as biexciton formation or a nonradiative Auger process. In addition, the LET dependence was most pronounced for $\left(\mathrm{C}_{6} \mathrm{H}_{5} \mathrm{C}_{2} \mathrm{H}_{4} \mathrm{NH}_{3}\right)_{2} \mathrm{PbBr}_{4}$, whose radiative rate and luminescence quantum efficiency were the highest among the investigated compounds. This result is because the radiative process in this compound, as a major decay process, is more significantly influenced by excited state interactions at higher LETs.

\section{Introduction}

It is widely known that high-energy heavy charged particles densely lose their energy in condensed matter. The one-dimensional density of the energy deposition along the trajectories of particles, i.e., linear energy transfer (LET), has long been used as a quantity for describing the characteristics of dense energy deposition. The dependence of the irradiation effects of solids on the LET has been widely investigated, ${ }^{(1)}$ and several types of irradiation effects have been used for processing materials, such as the formation of columnar defects as pinning centers in high-temperature oxide superconductors ${ }^{(2)}$ and ion-track etching in polymers. ${ }^{(3)}$

In the case of radiation detection, the LET dependence of the detector response has long been identified as an issue. The LET dependence of the response of scintillators was phenomenologically formulated by Birks as early as $1964 .^{(4)}$ This formula has long been used for compensating the LET-dependent scintillation light yield, although the formula has little physical basis and limited applicability. From a technical viewpoint, the LET-dependent

*Corresponding author: e-mail: koshi@qpc.che.tohoku.ac.jp

https://doi.org/10.18494/SAM.2021.3314 
response hinders accurate measurement of the deposition energy of heavy charged particles in scintillation detectors. In contrast, in the case of thermal neutron detection via nuclear reactions such as ${ }^{6} \mathrm{Li}(\mathrm{n}, \alpha){ }^{3} \mathrm{H}$ and $\left.{ }^{10} \mathrm{~B}(\mathrm{n}, \alpha)\right)^{7} \mathrm{Li}$, reaction products such as $\alpha$-rays, ${ }^{3} \mathrm{H}$, and ${ }^{7} \mathrm{Li}$ deposit their energy at a higher LET than that of X-rays or gamma rays. For scintillators whose rise and decay behavior significantly depends on the LET, detection events of thermal neutrons can be discriminated from those of gamma rays on the basis of a difference in the detection signal shape. ${ }^{(5)}$

LET-dependent scintillation properties are caused by the interaction of excited states. Because excited states are mobile and their density decreases during diffusion, the LET dependence and the excited state interactions responsible for this dependence have to be analyzed from the viewpoint of the dynamics of scintillation, i.e., scintillation temporal profiles. Previously, Kimura and coworkers measured the LET-dependent scintillation decay of $\mathrm{BaF}_{2}$ using their own system based on a time-correlated single photon counting technique. ${ }^{(6-8)}$ Recently, we have constructed a measurement system of LET-dependent scintillation decay based on pulsed ion beams. ${ }^{(9)}$ Thus far, we have reported the LET-dependent scintillation rise and decay of scintillators having dopants as luminescent centers, such as Ce-doped Li-glass, ${ }^{(10)}$ Ce-doped $\mathrm{LiCaAlF}_{6},{ }^{(11)} \mathrm{Ce}$-doped $\mathrm{Gd}_{2} \mathrm{SiO}_{5},{ }^{(12)}$ and Ce-doped $\mathrm{Gd}_{3} \mathrm{Al}_{2} \mathrm{Ga}_{3} \mathrm{O}_{12}{ }^{(13)}$ scintillators, in which competition between the excitation energy transfers from the host to luminescent centers and quenching due to excited state interactions have been observed. Also, we have reported the LET-dependent scintillation rise and decay of self-activated scintillators such as $\mathrm{Bi}_{4} \mathrm{Ge}_{3} \mathrm{O}_{12},{ }^{(14)}$ in which faster rise and decay were observed at higher LETs and attributed to the interactions of self-trapped excitons nearby.

In this study, we focused on the LET dependence of the scintillation properties of a novel class of scintillators, i.e., organic-inorganic layered perovskite-type compounds. The basic concept of scintillator development based on these compounds was proposed by our group more than 15 years ago. ${ }^{(15-17)}$ Recently, we have developed single-crystalline scintillators based on compounds with a relatively high light yield and fast decay. ${ }^{(18-21)}$ These compounds have a layered structure of alternating organic amine layers and inorganic lead halide layers. Owing to the significantly lower band-gap energy of the inorganic layers than that of the organic layers, the compounds have multiple quantum-well structures in their crystal structure. Fast and efficient scintillation has successfully been obtained from quantum-confined Wannier excitons in the inorganic layers. ${ }^{(16,17)}$ In this study, we analyzed the LET dependence of the scintillation properties of such compounds with a focus on the scintillation temporal profiles from the viewpoint of interactions of Wannier excitons at higher LETs.

\section{Materials and Methods}

The samples were single crystals of layered perovskite-type compounds with phenethylamine, benzylamine, or butylamine in their organic layers. The chemical formulae of these compounds were $\left(\mathrm{C}_{6} \mathrm{H}_{5} \mathrm{C}_{2} \mathrm{H}_{4} \mathrm{NH}_{3}\right)_{2} \mathrm{PbBr}_{4},\left(\mathrm{C}_{6} \mathrm{H}_{5} \mathrm{CH}_{2} \mathrm{NH}_{3}\right)_{2} \mathrm{PbBr}_{4}$, and $\left(\mathrm{C}_{4} \mathrm{H}_{9} \mathrm{NH}_{3}\right)_{2} \mathrm{PbBr}_{4}$ and are respectively denoted as Phe, Ben, and $\mathrm{C} 4$ hereafter. The compounds were synthesized through the reaction of the amine and hydrobromic acid, and subsequent reaction of the reaction 
products with $\mathrm{PbBr}_{2}$. Single crystals were grown using a poor solvent diffusion method. ${ }^{(15-17)}$ The in-plane size of the sample crystals was approximately $1 \times 1 \mathrm{~cm}^{2}$. The thicknesses of the crystals were $0.95,0.10$, and $0.25 \mathrm{~mm}$ for Phe, Ben, and $\mathrm{C} 4$, respectively. The details of the sample preparation procedures were reported in previous papers. ${ }^{(19-21)}$

The scintillation temporal profiles at different LETs were measured using a measurement system at TIARA, QST, Japan. We used pulsed ion beams from an azimuthally varying field (AVF) cyclotron as the excitation source. The samples were irradiated with pulsed beams of $20 \mathrm{MeV} \mathrm{H}^{+}, 50 \mathrm{MeV} \mathrm{He}{ }^{2+}$, and $220 \mathrm{MeV} \mathrm{C}^{5+}$ from the AVF cyclotron. The pulsed beams were generated using a pair of choppers. The details of the pulsed beam generation method were reported in a previous paper. ${ }^{(2)}$ The samples were irradiated with the pulsed ion beams in air in an irradiation room. The scintillation from the samples was detected with a photomultiplier tube (PMT; Hamamatsu R7400U). The detection signals of the PMT were delivered from the irradiation room to another room in which the signals were recorded using a digital oscilloscope (DSO; Tektronix DPO 7104). The trigger signals synchronized with the pulsed ion beams for the DSO were supplied from the accelerator. The detection signals were averaged over 1000 pulses to obtain a scintillation temporal profile. The origin of the time was determined as the maximum in the scintillation temporal profiles of a plastic scintillator (BC-400) using the same setup of the measurement system. The overall time resolution of the measurement system was $\sim 2 \mathrm{~ns}$ at half width at half maximum (HWHM). The details of the measurement system were reported in a previous paper. ${ }^{(9)}$

\section{Results and Discussion}

Figure 1 depicts the LETs of $20 \mathrm{MeV} \mathrm{H}^{+}, 50 \mathrm{MeV} \mathrm{He}^{2+}$, and $220 \mathrm{MeV} \mathrm{C}^{5+}$ for the samples, estimated using the SRIM code. ${ }^{(23)}$ Under the experimental conditions of this study, the LET was higher for the heavier ions in all samples.

Figures 2-4 present the scintillation temporal profiles up to $50 \mathrm{~ns}$ of Phe, Ben, and C4 for the different ions, respectively. The maximum of the scintillation temporal profiles is slightly different for the different ions. We determined the origin of the time axis as the peak time of the scintillation temporal profiles of a commercially available BC-400 plastic scintillator, as explained in Sect. 2. The slight difference originates from the error in the determination of the peak time. The LET dependence of the rise in the scintillation temporal profile was negligible for all the samples. The HWHM of the rise was approximately 2-3 ns and comparable to the time resolution of the measurement system. Hence, we cannot exclude the possibility of a LETdependent rise within the time resolution. The initial decay up to $50 \mathrm{~ns}$, however, exhibited a complicated LET dependence, particularly for Phe: the fastest and slowest decays were observed for irradiation of $220 \mathrm{MeV} \mathrm{C}^{5+}$ and $50 \mathrm{MeV} \mathrm{He}^{2+}$, respectively, i.e., the decay became slower in the LET range from $20 \mathrm{MeV} \mathrm{H}^{+}$to $50 \mathrm{MeV} \mathrm{He}^{2+}$, and subsequently became faster in the LET range from $50 \mathrm{MeV} \mathrm{He}^{2+}$ to $220 \mathrm{MeV} \mathrm{C}^{5+}$. The typical error in the measurements is within the width of the lines presented in Figs. 2-4. The difference in the initial decay (0-20 ns) of the temporal profiles of different ions is larger than the line width for Phe and Ben, although the difference is comparable to the line width for $\mathrm{C} 4$. The LET dependence was most pronounced 

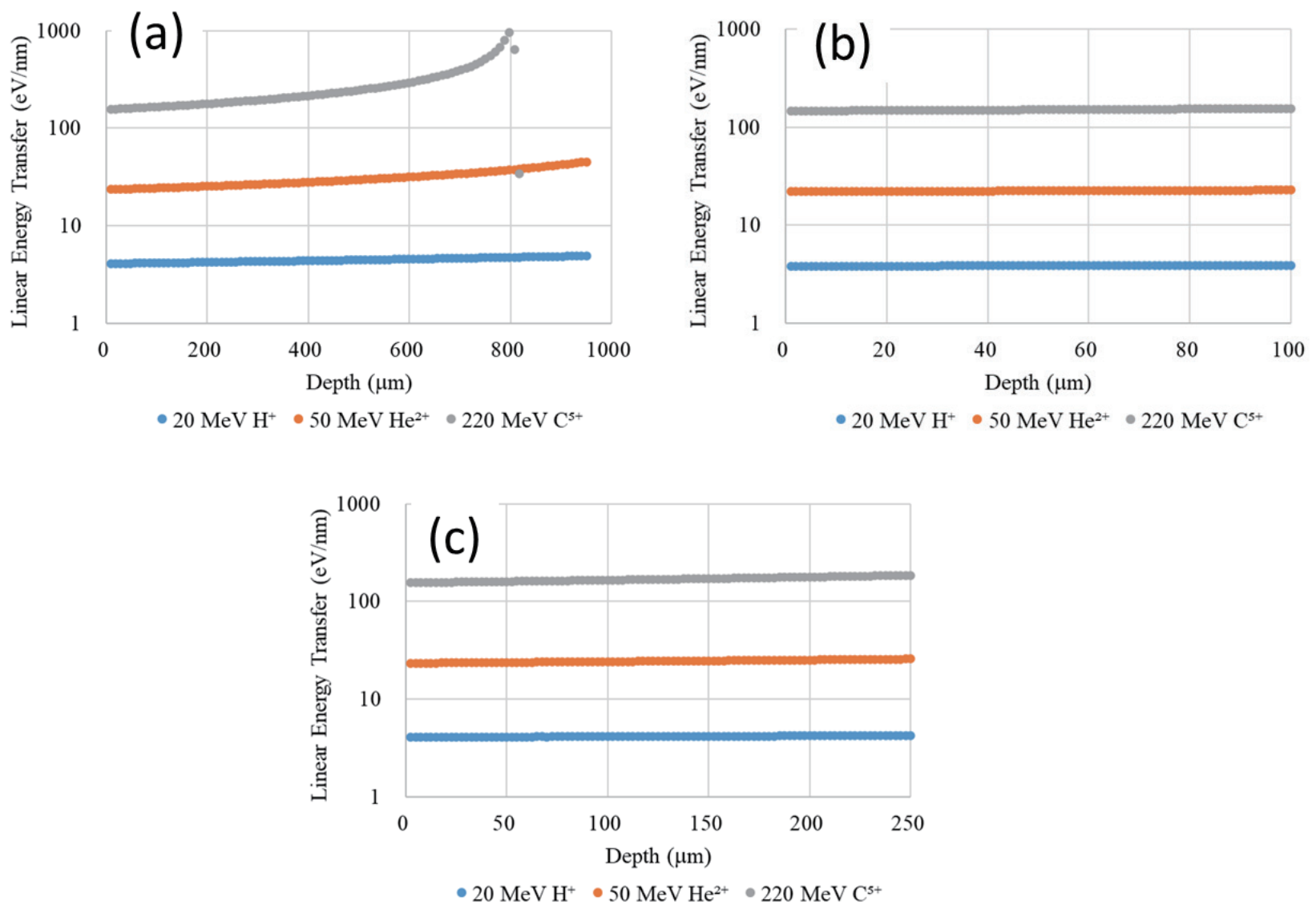

Fig. 1. (Color online) LETs of (a) $\left(\mathrm{C}_{6} \mathrm{H}_{5} \mathrm{C}_{2} \mathrm{H}_{4} \mathrm{NH}_{3}\right)_{2} \mathrm{PbBr}_{4}$ (Phe), (b) $\left(\mathrm{C}_{6} \mathrm{H}_{5} \mathrm{CH}_{2} \mathrm{NH}_{3}\right)_{2} \mathrm{PbBr}_{4}$ (Ben), and $\left(\mathrm{C}_{4} \mathrm{H}_{9} \mathrm{NH}_{3}\right)_{2} \mathrm{PbBr}_{4}(\mathrm{C} 4)$, estimated using the SRIM code. ${ }^{(23)}$

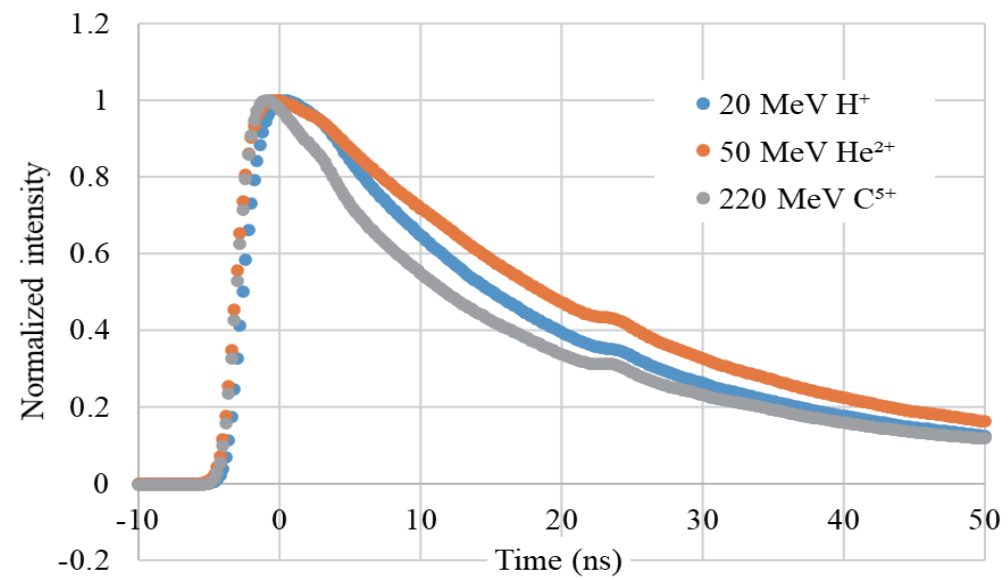

Fig. 2. (Color online) Scintillation temporal profiles up to $50 \mathrm{~ns}$ of $\left(\mathrm{C}_{6} \mathrm{H}_{5} \mathrm{C}_{2} \mathrm{H}_{4} \mathrm{NH}_{3}\right)_{2} \mathrm{PbBr}_{4}$ (Phe) under irradiation with $20 \mathrm{MeV} \mathrm{H}^{+}, 50 \mathrm{MeV} \mathrm{He}^{2+}$, and $220 \mathrm{MeV} \mathrm{C}^{5+}$. 


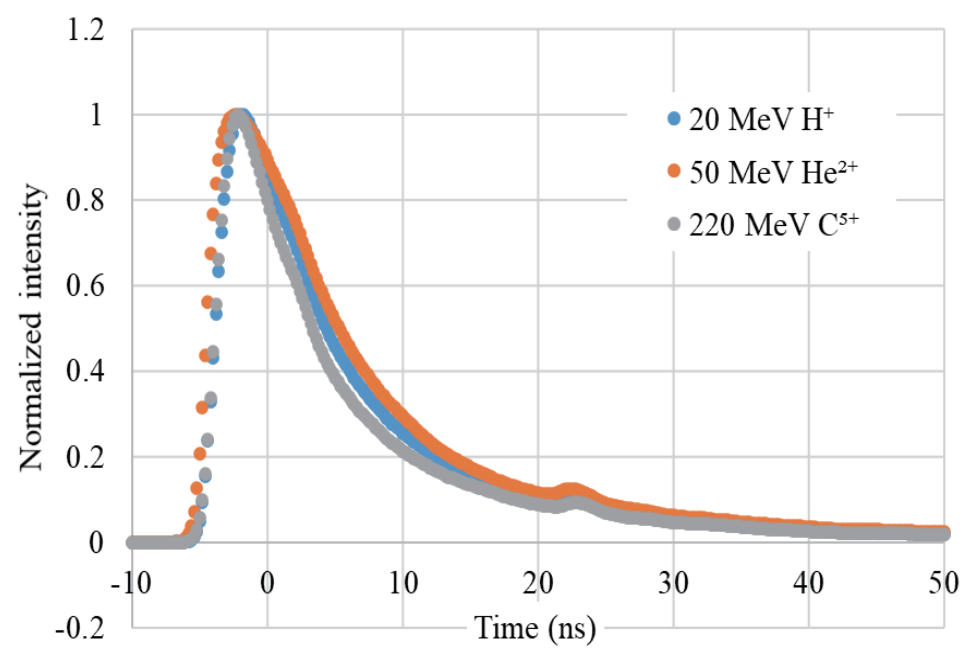

Fig. 3. (Color online) Scintillation temporal profiles up to $50 \mathrm{~ns}$ of $\left(\mathrm{C}_{6} \mathrm{H}_{5} \mathrm{CH}_{2} \mathrm{NH}_{3}\right)_{2} \mathrm{PbBr}_{4}$ (Ben) under irradiation with $20 \mathrm{MeV} \mathrm{H}^{+}, 50 \mathrm{MeV} \mathrm{He}^{2+}$, and $220 \mathrm{MeV} \mathrm{C}^{5+}$.

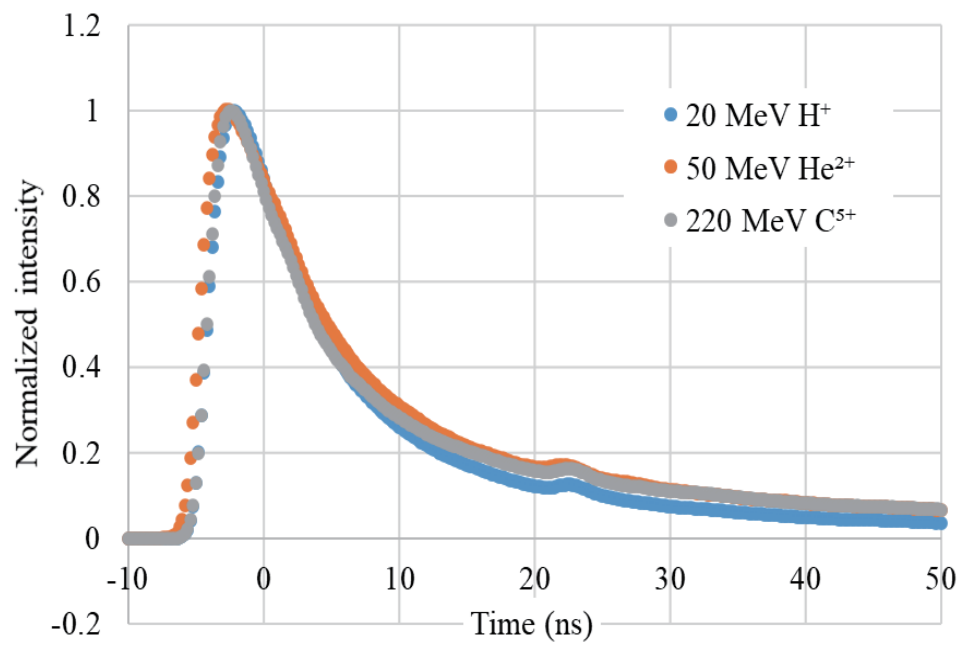

Fig. 4. (Color online) Scintillation temporal profiles up to $50 \mathrm{~ns}$ of $\left(\mathrm{C}_{4} \mathrm{H}_{9} \mathrm{NH}_{3}\right)_{2} \mathrm{PbBr}_{4}(\mathrm{C} 4)$ under irradiation with $20 \mathrm{MeV} \mathrm{H}^{+}, 50 \mathrm{MeV} \mathrm{He}^{2+}$, and $220 \mathrm{MeV} \mathrm{C}^{5+}$.

for Phe, and a significant LET dependence was also observed for Ben. No significant LET dependence was observed for $\mathrm{C} 4$.

Figures 5-7 illustrate the scintillation temporal profiles up to $400 \mathrm{~ns}$ of Phe, Ben, and C4 for the different ions, respectively. Some significant noise features can be seen at 120, 180, 210, and $240 \mathrm{~ns}$ and are attributed to electrical noise derived from reflection of the detection signals. Nevertheless, the LET-dependent decay behavior on a long-term scale can be discussed on the basis of the obtained results. However, the LET dependence was quite complicated: the fastest and slowest decays were for $220 \mathrm{MeV} \mathrm{C}^{5+}$ and $50 \mathrm{MeV} \mathrm{He}^{2+}$, respectively, which is the same 


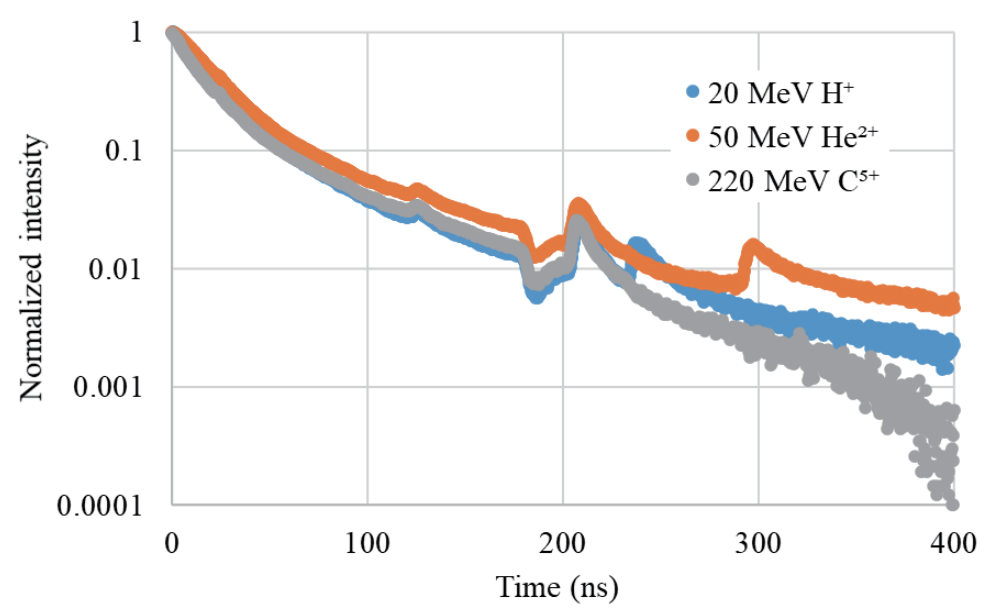

Fig. 5. (Color online) Scintillation temporal profiles up to $400 \mathrm{~ns}$ of $\left(\mathrm{C}_{6} \mathrm{H}_{5} \mathrm{C}_{2} \mathrm{H}_{4} \mathrm{NH}_{3}\right)_{2} \mathrm{PbBr}_{4}$ (Phe) under irradiation with $20 \mathrm{MeV} \mathrm{H}^{+}, 50 \mathrm{MeV} \mathrm{He}^{2+}$, and $220 \mathrm{MeV} \mathrm{C}^{5+}$.

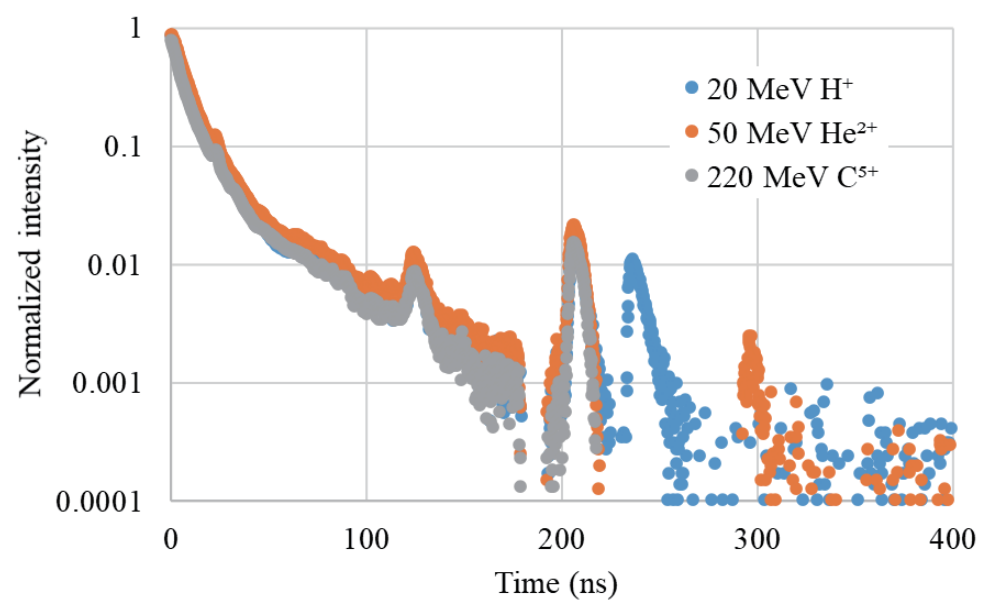

Fig. 6. (Color online) Scintillation temporal profiles up to $400 \mathrm{~ns}$ of $\left(\mathrm{C}_{6} \mathrm{H}_{5} \mathrm{CH}_{2} \mathrm{NH}_{3}\right)_{2} \mathrm{PbBr}_{4}$ (Ben) under irradiation with $20 \mathrm{MeV} \mathrm{H}^{+}, 50 \mathrm{MeV} \mathrm{He}^{2+}$, and $220 \mathrm{MeV} \mathrm{C}^{5+}$.

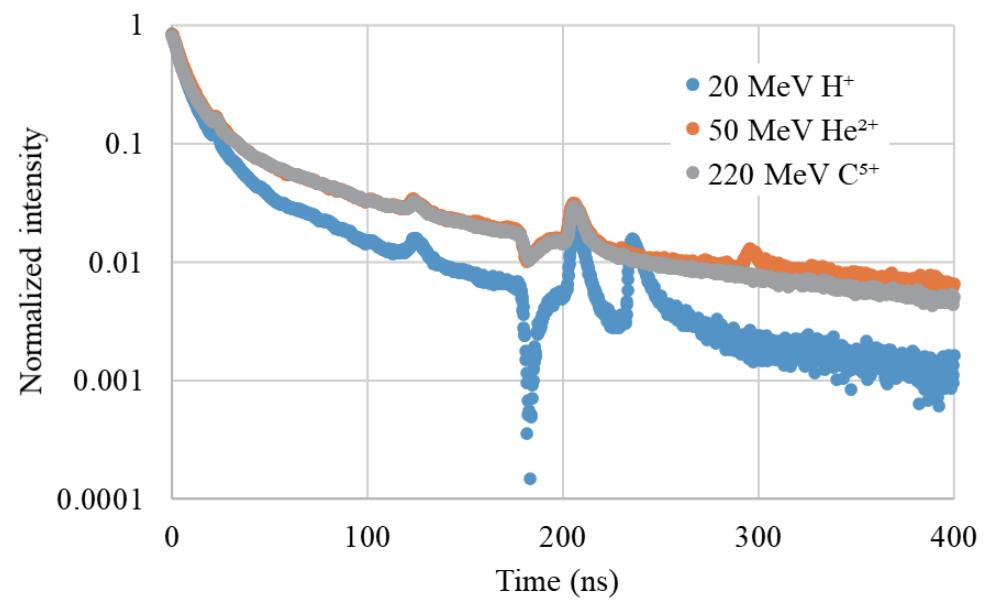

Fig. 7. (Color online) Scintillation temporal profiles up to $400 \mathrm{~ns}$ of $\left(\mathrm{C}_{4} \mathrm{H}_{9} \mathrm{NH}_{3}\right)_{2} \mathrm{PbBr}_{4}(\mathrm{C} 4)$ under irradiation with $20 \mathrm{MeV} \mathrm{H}^{+}, 50 \mathrm{MeV} \mathrm{He}^{2+}$, and $220 \mathrm{MeV} \mathrm{C}^{5+}$. 
trend as seen for the initial decay behavior. For Ben, we observed a negligible LET dependence apart from in the initial decay behavior. Among the scintillation temporal profiles of $\mathrm{C} 4$, the fastest decay was observed for $20 \mathrm{MeV} \mathrm{H}^{+}$irradiation, which is not consistent with the LET dependence of Phe.

The decay behavior up to $150 \mathrm{~ns}$, which did not include the significant afterpulse of the PMT at around $180 \mathrm{~ns}$, can be fitted with a sum of two exponential decay functions. Table 1 summarizes the decay time constants and the proportion of the first component. The LET dependence is clear in this table: the decay was the slowest for $50 \mathrm{MeV} \mathrm{He}^{2+}$ for all samples. The decay time constants of the first component were longer than those under X-ray irradiation of 9.6, 2.8, and $1.1 \mathrm{~ns}$ for Phe, Ben, and C4, respectively. ${ }^{(20)}$ This is because the time resolution of the measurement system using pulsed ion beams is comparable to the decay time constants. ${ }^{(13)}$

To explain the initial decay process, we need to discuss the dynamics of free excitons, because the fast component has been attributed to free excitons. ${ }^{(19-21)}$ For the faster decay at higher LETs or excitation densities, some processes can be proposed: one is the formation of biexcitons, whose rate of the radiative process, i.e., exciton recombination with the emission of an optical photon, is significantly higher than that of free excitons. ${ }^{(24)}$ Another possible cause of the faster decay at higher excitation densities is the nonradiative Auger process, in which one exciton during its annihilation gives its energy to another exciton nearby, which is converted into a higher excited state. ${ }^{(25)}$ The competition of the Auger process with the radiative process of free excitons results in fast decay. In contrast, little has been published on slower decay at higher excitation densities. A possible cause of this excitation-density dependence is the competition between the radiative process and trapping at nonradiative sites: at high excitation densities, some excitons can survive trapping at nonradiative sites at which other excitons have already been trapped. Regarding the competition between multiple excited states for a trapping site, we have observed a deceleration process in the energy transfer of Ce-doped $\mathrm{LiCaAlF}_{6}$ at high LETs and attributed this to competition, in which an excited state in the host matrix cannot transfer its energy to luminescent centers already in the excited state at high excitation densities. ${ }^{(1)}$ As summarized in Table 1, the decay time constant of the first component became longer with increasing LET from $20 \mathrm{MeV} \mathrm{H}^{+}$to $50 \mathrm{MeV} \mathrm{He}^{2+}$, which is attributed to the

Table 1

Decay time constants and proportion of the first component.

\begin{tabular}{lcccc}
\hline Sample & Ion beam & $\begin{array}{c}\text { Decay time constant of } \\
\text { first component (ns) }\end{array}$ & $\begin{array}{c}\text { Decay time constant of } \\
\text { second component (ns) }\end{array}$ & $\begin{array}{c}\text { Proportion of first } \\
\text { component (\%) }\end{array}$ \\
\hline \multirow{3}{*}{ Phe } & $20 \mathrm{MeV} \mathrm{H}^{+}$ & 21.7 & 290 & 67 \\
& $50 \mathrm{MeV} \mathrm{He}^{2+}$ & 25.9 & 2527 & 26 \\
& $220 \mathrm{MeV} \mathrm{C}^{5+}$ & 13.5 & 54.8 & 43 \\
\multirow{3}{*}{ Ben } & $20 \mathrm{MeV} \mathrm{H}^{+}$ & 7.7 & 38.0 & 73 \\
& $50 \mathrm{MeV} \mathrm{He}^{2+}$ & 9.1 & 60.8 & 78 \\
& $220 \mathrm{MeV} \mathrm{C}^{5+}$ & 6.5 & 40.5 & 64 \\
$\mathrm{C} 4$ & $20 \mathrm{MeV} \mathrm{H}^{+}$ & 7.1 & 45.7 & 54 \\
& $50 \mathrm{MeV} \mathrm{He}^{2+}$ & 8.1 & 68.9 & 42 \\
& $220 \mathrm{MeV} \mathrm{C}^{5+}$ & 6.5 & 57.6 & 32 \\
\hline
\end{tabular}


competition between the radiative process and trapping at nonradiative sites. The shorter decay time constants for $220 \mathrm{MeV} \mathrm{C}^{5+}$ than those of $50 \mathrm{MeV} \mathrm{He}^{2+}$ can be attributed to the nonradiative Auger process.

The LET dependence of the initial decay was most pronounced for Phe. This result can be discussed on the basis of the difference in the radiative rates and luminescence quantum efficiencies of Phe, Ben, and C4: the radiative rate and quantum efficiency are in the order Phe $>$ Ben $>$ C4. According to our previous paper, ${ }^{(19)}$ the quantum efficiencies of Phe, Ben, and $\mathrm{C} 4$ were $22 \%, 3.8 \%$, and $0.36 \%$, and their radiative decay rates were $4.3 \times 10^{7}, 9.9 \times 10^{6}$, and $2.2 \times$ $10^{6} \mathrm{~s}^{-1}$, respectively. For Phe, the radiative process plays a major role in the decay, and its decay process can be significantly influenced by the excited state interaction at higher LETs. On the other hand, for $\mathrm{C} 4$, the radiative process plays only a minor role and it is thus less influenced by the excited state interaction at higher LETs.

Regarding the LET dependence of the decay on a longer time scale, the decay component can be attributed to the emission of trapped excitons. ${ }^{(19-21)}$ Trapping sites, such as defects or impurities, are involved in the decay process. The LET-dependent decay on this time scale is influenced by the excited state interaction and trapping at trapping sites, and thus shows complicated behavior. At present, a straightforward explanation of the observed LET dependence is difficult.

\section{Conclusions}

We observed the temporal scintillation profiles of organic-inorganic layered hybrid compounds at different LETs. The LET dependence of the initial decay rate was complicated: the decay was initially slower and subsequently became faster for higher LETs in the LET range investigated in this study. The faster decay at higher LETs can be explained in terms of the interactions of excitons such as biexciton formation or a nonradiative Auger process. In contrast, the slower decay at higher LETs can be explained in terms of the competition between the radiative process and trapping at nonradiative sites. At high excitation densities, some excitons can survive trapping at nonradiative sites at which other excitons have already been trapped. The LET dependence was more pronounced for compounds with higher radiative rates and luminescence quantum efficiencies, which indicates that the excited state interactions at higher LETs have a greater influence on the radiative process in compounds for which the radiative process plays a more important role in their decay.

\section{Acknowledgments}

This research was supported by a Grant-in-Aid for Scientific Research (A) (No. 18H03890, 2018-2021) and a Grant-in-Aid for Scientific Research (B) (No. 18H01911, 2018-2020). Part of this research is based on the Cooperative Research Project of the Research Center for Biomedical Engineering, Ministry of Education, Culture, Sports, Science and Technology of Japan. 


\section{References}

1 F. F. Komarov: Phys. Usp. 60 (2017) 435.

2 L. Civale: Supercond. Sci. Technol. 10 (1997) A11.

3 F. Liu, M. Wang, X. Wang, P. Wang, W. Shen, S. Ding, and Y. Wang: Nanotechnology 30 (2019) 052001.

4 J. B. Birks: The Theory and Practice of Scintillation Counting (Pergamon, New York, 1964).

5 A. R. Spowart: J. Phys. C: Solid State Phys. 12 (1979) 3369.

6 K. Kimura: J. Electron Spectrosc. Related Phenomena 79 (1996) 43.

7 K. Kimura: Nucl. Instrum. Methods Phys. Res., Sect. B 116 (1996) 57.

8 K. Kimura and W. Hong: Phys. Rev. B 58 (1998) 6081.

9 M. Koshimizu, S. Kurashima, M. Taguchi, K. Iwamatsu, A. Kimura, and K. Asai: Rev. Sci. Instr. 86 (2015) 013101.

10 M. Koshimizu, K. Iwamatsu, M. Taguchi, S. Kurashima, A. Kimura, T. Yanagida, Y. Fujimoto, K. Watanabe, and K. Asai: J. Lumin. 169 (2016) 678.

11 T. Yanagida, M. Koshimizu, S. Kurashima, K. Iwamatsu, A. Kimura, M. Taguchi, Y. Fujimoto, and K. Asai: Nucl. Instrum. Methods Phys. Res., Sect. B 365 (2015) 529.

12 T. Yanagida, M. Koshimizu, Y. Fujimoto, S. Kurashima, K. Iwamatsu, A. Kimura, M. Taguchi, G. Okada, and N. Kawaguchi: Nucl. Instrum. Methods Phys. Res., Sect. B 409 (2017) 27.

13 M. Koshimizu, S. Kurashima, A. Kimura, M. Taguchi, T. Yanagida, H. Yagi, T. Yanagitani, Y. Fujimoto, and K. Asai: Nucl. Instrtrum. Methods Phys. Res., Sect. B 471 (2020) 59.

14 M. Koshimizu, S. Kurashima, A. Kimura, M. Taguchi, T. Yanagida, Y. Fujimoto, and K. Asai: Nucl. Instrum. Methods Phys. Res., Sect. B 409 (2017) 19.

15 K. Shibuya, M. Koshimizu, Y. Takeoka, and K. Asai: Nucl. Instrum. Methods Phys. Res., Sect. B 194 (2002) 207.

16 K. Shibuya, M. Koshimizu, K. Asai, and H. Shibata: Appl. Phys. Lett. 84 (2004) 4370.

17 K. Shibuya, M. Koshimizu, H. Murakami, Y. Muroya, Y. Katsumura, and K. Asai: Jpn. J. Appl. Phys. 43 (2004) L1333.

18 S. Kishimoto, K. Shibuya, F. Nishikido, M. Koshimizu, R. Haruki, and Y. Yoda: Appl. Phys. Lett. 93 (2008) 261901.

19 N. Kawano, M. Koshimizu, Y. Sun, N. Yahaba, Y. Fujimoto, T. Yanagida, and K. Asai: J. Phys. Chem. C 118 (2014) 9101.

20 N. Kawano, M. Koshimizu, A. Horiai, F. Nishikido, R. Haruki, S. Kishimoto, K. Shibuya, Y. Fujimoto, T. Yanagida, and K. Asai: Jpn. J. Appl. Phys. 55 (2016) 110309.

21 N. Kawano, M. Koshimizu, G. Okada, Y. Fujimoto, N. Kawaguchi, T. Yanagida, and K. Asai: Sci. Rep. 7 (2017) 14754.

22 S. Kurashima, N. Miyawaki, H. Kashiwagi, S. Okumura, M. Taguchi, and M. Fukuda: Rev. Sci. Instrum. 86 (2015) 073311.

23 J. F. Ziegler, J. P. Biersack, and U. Littmark: The Stopping and Range of Ions in Solids (Pergamon Press, New York, 1984).

24 Y. Yamamoto, G. Oohata, K. Mizoguchi, H. Ichida, and Y. Kanematsu: Phys. Status Solidi C 9 (2012) 2501.

25 L. M. Herz: Ann. Rev. Phys. Chem. 67 (2016) 65. 